\title{
Optimally-Tuned Nonparametric Linear Equalization for Massive MU-MIMO Systems
}

\section{Conference Paper}

\section{Author(s):}

Ghods, Ramina; Jeon, Charles; Mirza, Gulnar; Maleki, Arian; Studer, Christoph (iD

Publication date:

2017

Permanent link:

https://doi.org/10.3929/ethz-b-000455584

Rights / license:

In Copyright - Non-Commercial Use Permitted

Originally published in:

https://doi.org/10.1109/ISIT.2017.8006903 


\title{
Optimally-Tuned Nonparametric Linear Equalization for Massive MU-MIMO Systems
}

\author{
Ramina Ghods, Charles Jeon, Gulnar Mirza, Arian Maleki, and Christoph Studer
}

\begin{abstract}
This paper deals with linear equalization in massive multi-user multiple-input multiple-output (MU-MIMO) wireless systems. We first provide simple conditions on the antenna configuration for which the well-known linear minimum meansquare error (L-MMSE) equalizer provides near-optimal spectral efficiency, and we analyze its performance in the presence of parameter mismatches in the signal and/or noise powers. We then propose a novel, optimally-tuned NOnParametric Equalizer (NOPE) for massive MU-MIMO systems, which avoids knowledge of the transmit signal and noise powers altogether. We show that NOPE achieves the same performance as that of the L-MMSE equalizer in the large-antenna limit, and we demonstrate its efficacy in realistic, finite-dimensional systems. From a practical perspective, NOPE is computationally efficient and avoids dedicated training that is typically required for parameter estimation.
\end{abstract}

\section{INTRODUCTION}

Massive (or large-scale) multi-user multiple-input multipleoutput (MU-MIMO) will be among the key technologies for fifth-generation $(5 \mathrm{G})$ wireless systems as it provides (often significantly) higher spectral efficiency than traditional, smallscale MIMO [1], [2]. Data detection at the infrastructure base-stations (BSs) in such massive MU-MIMO systems is among the most critical components from a spectral efficiency and computational complexity perspective. In particular, since optimal data detection is known to be an NP-hard problem [3], a naïve exhaustive search over all possible transmit signals would result in prohibitive computational complexity for such largedimensional systems. Hence, alternative algorithms that achieve high spectral efficiency at low complexity must be deployed in practice. In addition, practical massive MU-MIMO systems suffer-as do traditional MIMO systems-from real-world hardware impairments and model mismatches (for example amplifier nonlinearities, phase noise, quantization artifacts, channel-estimation errors, etc.). Such system nonidealities are known to substantially reduce the performance of optimal data-detection algorithms unless one explicitly models these impairments and estimates the associated parameters [4].

\section{A. Contributions}

In this paper, we address the above challenges by developing a novel, nonparametric equalizer (NOPE) for massive MUMIMO systems that requires low complexity and is robust

R. Ghods, C. Jeon, G. Mirza, and C. Studer are with the School of ECE at Cornell University, Ithaca, NY; e-mails: rg548@ cornell.edu, jeon@csl.cornell.edu,gzm3@cornell.edu, and studer@cornell.edu.

A. Maleki is with Department of Statistics at Columbia University, New York City, NY; e-mail: arian@stat.columbia.edu.

The work of RG, CJ, and CS was supported in part by Xilinx, Inc. and by the US National Science Foundation (NSF) under grants ECCS-1408006, CCF-1420328, and CAREER CCF-1652065. to system impairments and model mismatches. Our key contributions can be summarized as follows:

- We present simple conditions for which linear minimum mean-squared error (L-MMSE), zero-forcing (ZF), and maximum ratio combining (MRC)-based equalizers provide near-optimal performance in massive MU-MIMO systems.

- We analyze the impact of parameter mismatches on L-MMSE equalization by extending the results by Tse and Hanly in [5].

- We propose a novel, computationally efficient, and nonparametric algorithm called NOPE that does not require any knowledge of the signal and noise powers.

- We prove, in the large-antenna limit, that NOPE achieves the same performance as that of the L-MMSE equalizer, which requires knowledge of the signal and noise powers.

- We demonstrate that NOPE achieves the performance of the L-MMSE equalizer in realistic, finite-dimensional systems.

\section{B. Relevant Prior Art}

L-MMSE estimation is used in a large number of communication applications for estimation, detection, and equalization [5][10]. The low complexity (besides the inversion of a potentially large matrix) and acceptable performance in many situations are responsible for the widespread use of L-MMSE estimation in practical transceiver designs. For massive MU-MIMO systems, it was shown in [11] that L-MMSE equalization enables (often significantly) higher achievable rates than ZF or MRC-based equalizers. To complement these results, we provide conditions on the antenna configuration for which L-MMSE, ZF, and MRC approach the fundamental performance limits.

One of the downsides of L-MMSE equalization is that it requires accurate estimates of the signal and noise powers. While Al-Dhahir and Cioffi in [12] analyzed the effect of parameter mismatches to MMSE-based decision-feedback equalizers, we extend the results by Tse and Hanly in [5] and provide large-antenna limit expressions for the output signal-to-inference ratio (SIR) of L-MMSE equalization in the presence of parameter mismatches.

To mitigate the impact of parameter mismatches, linear and adaptive methods have been proposed for randomly spread code-division multiple-access (CDMA) systems in [13], [14]. Inspired by such results, we propose an optimally-tuned, nonparametric linear equalization algorithm for massive MUMIMO systems. Our algorithm does not require any knowledge of the signal and/or noise powers, achieves the performance of the L-MMSE equalizer in the large-antenna limit, and is computationally efficient as it avoids a costly matrix inversion. 


\section{Notation}

Lowercase and uppercase boldface letters designate column vectors and matrices, respectively. For a matrix $\mathbf{A}$, we define its conjugate transpose as $\mathbf{A}^{\mathrm{H}}$. The entry on the $k$-th row and $\ell$-th column is $A_{k, \ell}$, We define $\langle\mathbf{a}\rangle=\frac{1}{N} \sum_{k=1}^{N} a_{k}$. Multivariate complex-valued Gaussian probability density functions (PDFs) with mean vector $\mathbf{m}$ and covariance matrix $\mathbf{K}$ are denoted by $\mathcal{C N}(\mathbf{m}, \mathbf{K})$. Expectation and variance with respect to the PDF of the random variable $X$ is $\mathbb{E}_{X}[\cdot]$ and $\operatorname{Var}_{X}[\cdot]$, respectively.

\section{LINEAR MMSE EQUALIZATION}

We start by introducing the system model and reviewing the basics of parametric L-MMSE equalization with perfect knowledge of the signal and noise powers. We then develop a computationally efficient algorithm that relies on approximate message passing (AMP) and we analyze its performance.

\section{A. System Model}

We consider the following standard input-output relation of a massive MU-MIMO uplink system: $\mathbf{y}=\mathbf{H x}+\mathbf{n}$. Here, the vector $\mathbf{y} \in \mathbb{C}^{B}$ corresponds to the received signal ( $B$ denotes the number of BS antennas), the matrix $\mathbf{H} \in \mathbb{C}^{B \times U}$ represents the uplink channel ( $U$ denotes the number of user antennas), the transmit signal vector is $\mathbf{x} \in \mathbb{C}^{U}$, and the vector $\mathbf{n} \in \mathbb{C}^{B}$ models receive noise where all its entries are assumed to be i.i.d. circularly-symmetric complex Gaussian with variance $N_{0}$ per complex entry. We assume that the transmit signal vector $\mathbf{x}$ has i.i.d entries, i.e., $p(\mathbf{x})=\prod_{u=1}^{U} p\left(x_{u}\right)$, with zero mean and signal variance $E_{\mathrm{x}}=\mathbb{E}\left[\left|x_{u}\right|^{2}\right], \forall u$. We define the antenna ratio as $\beta=U / B$, and the following quantities:

Definition 1. The large-antenna limit is defined by fixing the antenna ratio $\beta=U / B$ and letting $U \rightarrow \infty$.

Definition 2. A matrix $\mathbf{H}$ has uniform channel gains if the entries of $\mathbf{H}$ are i.i.d. circularly-symmetric complex Gaussian with variance $1 / B$ per complex entry, i.e., $H_{b, u} \sim \mathcal{C N}(0,1 / B)$.

\section{B. Basics of L-MMSE Equalization}

We start with the following well-known facts. The equalization matrix $\mathbf{W} \in \mathbb{C}^{U \times B}$ of the L-MMSE equalizer output $\hat{\mathbf{x}}=\mathbf{W y}$ that minimizes the $M S E=\mathbb{E}_{\mathbf{x}, \mathbf{n}}\left[\|\hat{\mathbf{x}}-\mathbf{x}\|^{2}\right]$ is given by $\mathbf{W}=\left(\mathbf{H}^{\mathrm{H}} \mathbf{H}+N_{0} / E_{\mathrm{x}}\right)^{-1} \mathbf{H}^{\mathrm{H}}$. At high signalto-noise-ratio (SNR), i.e., for $N_{0} / E_{\mathrm{x}} \rightarrow 0$, the L-MMSE equalizer implements zero-forcing (ZF) equalization with output $\hat{\mathbf{x}}=\left(\mathbf{H}^{\mathrm{H}} \mathbf{H}\right)^{-1} \mathbf{H}^{\mathrm{H}} \mathbf{y}$; at low SNR, the L-MMSE equalizer implements MRC with output $\hat{\mathbf{x}}=\mathbf{H}^{\mathrm{H}} \mathbf{y}$. We note that L-MMSE is the optimal (linear and nonlinear) equalizer if the signal and noise are independent and both i.i.d. circularly symmetric complex Gaussian vectors.

\section{L-MMSE Equalization via AMP}

L-MMSE equalization as summarized above, can be implemented using the mismatched complex bayesian AMP (mcBAMP) algorithm proposed in [15], which is a mismatched version of the AMP algorithm put forward in [16]. In particular, we use mcB-AMP with the following Gaussian mismatched signal prior distribution: $\tilde{p}(\mathbf{x})=\prod_{i=1}^{N} \tilde{p}\left(x_{i}\right)$ with $\tilde{p}\left(x_{i}\right) \sim \mathcal{C N}\left(0, E_{\mathrm{x}}\right)$. By assuming that the detector knows the true transmit signal prior distribution $X_{0} \sim p\left(x_{0}\right)$ (e.g., two Dirac delta functions for BPSK), the parametric L-MMSE algorithm, which we will refer to as MMSE-AMP, is as follows:

Algorithm 1. Initialize $t=1, x_{\ell}^{1}=\mathbb{E}_{X_{0}}\left[X_{0}\right], \ell=1, \ldots, U$, and $\mathbf{r}^{1}=\mathbf{y}-\mathbf{H x}^{1}$. Then, for every iteration $t=1,2, \ldots, t_{\max }$ compute the output $\mathbf{z}^{t}$ via the following steps:

$$
\begin{aligned}
\tilde{\sigma}_{t}^{2} & =\frac{1}{B}\left\|\mathbf{r}^{t}\right\|_{2}^{2} \\
\tau^{t} & =\arg \min _{\tau \geq 0} \Psi\left(\tilde{\sigma}_{t}^{2}, \tau\right) \\
\mathbf{z}^{t} & =\mathbf{x}^{t}+\mathbf{H}^{\mathrm{H}} \mathbf{r}^{t} \\
\mathbf{x}^{t+1} & =\mathrm{F}^{m m}\left(\mathbf{z}^{t}, \tau^{t}\right) \\
\mathbf{r}^{t+1} & =\mathbf{y}-\mathbf{H} \mathbf{x}^{t+1}+\beta \mathbf{r}^{t}\left\langle\mathrm{~F}^{\prime m m}\left(\mathbf{z}^{t}, \tau^{t}\right)\right\rangle,
\end{aligned}
$$

Here, $\mathrm{F}^{m m}\left(x_{\ell}, \tau\right)=\frac{E_{\mathrm{x}}}{E_{\mathrm{x}}+\tau} x_{\ell}$ is the posterior mean function, $\mathrm{F}^{\prime m m}\left(x_{\ell}, \tau\right)$ is its derivative in the first argument, and both functions operate element-wise on vectors. Furthermore,

$\Psi\left(\tilde{\sigma}_{t}^{2}, \tau\right)=\mathbb{E}_{X_{0}, Z}\left[\left|\mathrm{~F}^{m m}\left(X_{0}+\tilde{\sigma}_{t} Z, \tau\right)-X_{0}\right|^{2}\right]=\frac{\tau^{2} E_{\mathrm{x}}+\sigma_{t}^{2} E_{\mathrm{x}}^{2}}{\left(E_{\mathrm{x}}+\tau\right)^{2}}$

is the MSE function in which the expectation is taken with respect to the true signal prior $X_{0} \sim p\left(x_{0}\right)$ and $Z \sim \mathcal{C N}(0,1)$.

We note that Algorithm 1 avoids the computation of matrix inverses, which often dominate the computational complexity of L-MMSE equalizers in small and large MIMO systems [17].

\section{Asymptotic Performance Analysis}

One of the key properties of AMP-based algorithms is that their SIR performance can be analyzed in the large-antenna limit using the state evolution (SE) framework. We require the following result; the proof follows from [15, Eq. 12]:

Lemma 1. Fix $\beta$ and let $\mathbf{H}$ have uniform channel gains. Then, in the large-antenna limit, the output $\mathrm{z}^{t}$ of Algorithm 1 can be modeled as $\mathbf{z}^{t}=\mathbf{x}+\mathbf{w}^{t}$ with $\mathbf{w}^{t} \sim \mathcal{C N}\left(0, \sigma_{t}^{2} \mathbf{I}_{U}\right)$, where the equivalent noise variance $\sigma_{t}^{2}$ in iteration $t$ is given by the following SE equation: $\sigma_{t+1}^{2}=N_{0}+\beta \frac{E_{\mathrm{x}}}{E_{\mathrm{x}}+\sigma_{t}^{2}} \sigma_{t}^{2}$.

For $t \rightarrow \infty$, the SE equation coincides with the asymptotic SIR expression of the L-MMSE equalizer given by Tse and Hanly in [5, Thm. 3.1] with $S I R=1 / \sigma^{2}$. Hence. we have:

Corollary 2. In the large-antenna limit, MMSE-AMP achieves the same SIR performance as the L-MMSE equalizer.

Since in the large-antenna limit and for uniform channel gains AMP-based equalization decouples the MIMO system into parallel and independent AWGN channels with variance $\sigma_{t}^{2}$ (see [18, Sec. 6] for the details of this decoupling property), the per-user achievable rate of the L-MMSE equalizer is given by $C_{\mathrm{L}-\mathrm{MMSE}}=\log _{2}\left(1+E_{\mathrm{x}} / \sigma^{2}\right)$ [bits/user/channel use], where $\sigma^{2}$ is the fixed-point to the SE equation in Lemma 1.

\section{E. When Does L-MMSE Achieve Near-Optimal Performance?}

As shown in [8], [9], [19] for massive MU-MIMO systems with significantly more BS antennas than users (i.e., for small values of $\beta$ ), linear equalizers, such as $\mathrm{MRC}, \mathrm{ZF}$, and $\mathrm{L}$ MMSE achieve near-optimal performance. More specifically, 


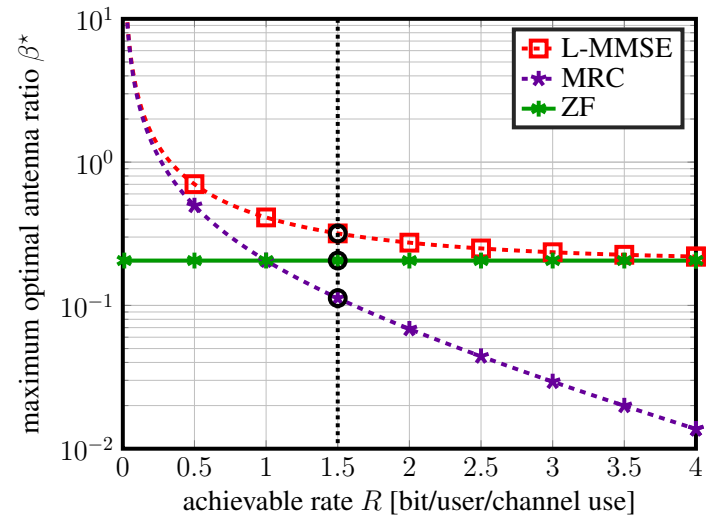

Fig. 1. Comparison of the maximum optimal antenna ratio (MOAR) $\beta^{\star}$ for MRC, ZF, and L-MMSE equalization to operate within $\delta \mathrm{SNR}=1 \mathrm{~dB}$ of the fundamental performance limit. For all achievable rates, L-MMSE supports the largest antenna ratio $\beta=U / B$ to operate within $1 \mathrm{~dB}$ of optimal performance.

reference [19] derives conditions for which ZF and MRC approach the performance of L-MMSE equalization. We now provide conditions on the antenna ratio $\beta$ for which linear equalizers approach optimal performance. We use the fact that, in the large-antenna limit, the individually-optimal (IO) posterior mean estimator (PME) decouples the MIMO system into $U$ parallel and independent AWGN channels $\mathbf{z}=\mathbf{x}+\mathbf{w}$ with $\mathbf{w} \sim \mathcal{C N}\left(0, \sigma^{2} \mathbf{I}_{U}\right)$, where the equivalent noise variance satisfies $\sigma^{2} \geq N_{0}$ [20]. Hence, the fundamental performance limit is given by the channel capacity of an AWGN channel $C_{\mathrm{AWGN}}=\log _{2}\left(1+E_{\mathrm{x}} / N_{0}\right)$. We therefore characterize the performance of linear equalizers as follows:

Definition 3. Assume that the IO-PME and a linear equalizer achieve the same rate $R$ at $S N R E_{\mathrm{x}} / N_{0}$ and $E_{\mathrm{x}} / \hat{N}_{0}$, respectively. We define the SNR loss as $\delta \mathrm{SNR}=N_{0} / \hat{N}_{0}$, which satisfies $1 \leq \delta \mathrm{SNR}$ and characterizes the excess SNR required by a linear equalizer to achieve optimal performance.

For MRC, ZF, and L-MMSE equalization, we can establish the following result with proof given in Appendix A-A.

Lemma 3. Assume the large-antenna limit and let $\mathbf{H}$ have uniform channel gains. For a fixed rate $R$, the SNR loss of $M R C, Z F$, and L-MMSE will be no larger than $\delta \mathrm{SNR}$ if $\beta \leq$ $\beta^{\star}(\delta \mathrm{SNR}, R)$, where $\beta^{\star}(\delta \mathrm{SNR}, R)$ is the maximum optimal antenna ratio (MOAR) given by

$$
\begin{aligned}
\text { MRC: } & \beta^{\star}(\delta \mathrm{SNR}, R)=\left(1-\delta \mathrm{SNR}^{-1}\right) \frac{1}{2^{R}-1} \\
\text { ZF: } & \beta^{\star}(\delta \mathrm{SNR}, R)=1-\delta \mathrm{SNR}^{-1} \\
L-M M S E: & \beta^{\star}(\delta \mathrm{SNR}, R)=\left(1-\delta \mathrm{SNR}^{-1}\right) \frac{2^{R}}{2^{R}-1} .
\end{aligned}
$$

Fig. 1 illustrates the MOAR for MRC, ZF, and L-MMSE equalization as a function of their achievable rates for a fixed SNR loss of $\delta \mathrm{SNR}=1 \mathrm{~dB}$. Lemma 3 identifies the maximum antenna ratio $\beta$ for which these linear equalizers are able to operate within $1 \mathrm{~dB}$ SNR of the fundamental performance limit. We see that L-MMSE enables the largest user-to-BS antenna ratios $\beta$ among the considered equalizers for all rates. Consider, for example, the dotted black vertical line at $1.5 \mathrm{bit} / \mathrm{user} / \mathrm{channel}$ use. We see that L-MMSE exhibits an SNR loss of less than $1 \mathrm{~dB}$ for any antenna ratio below 0.3; MRC and ZF require antenna ratios of 0.2 and 0.1 , respectively.

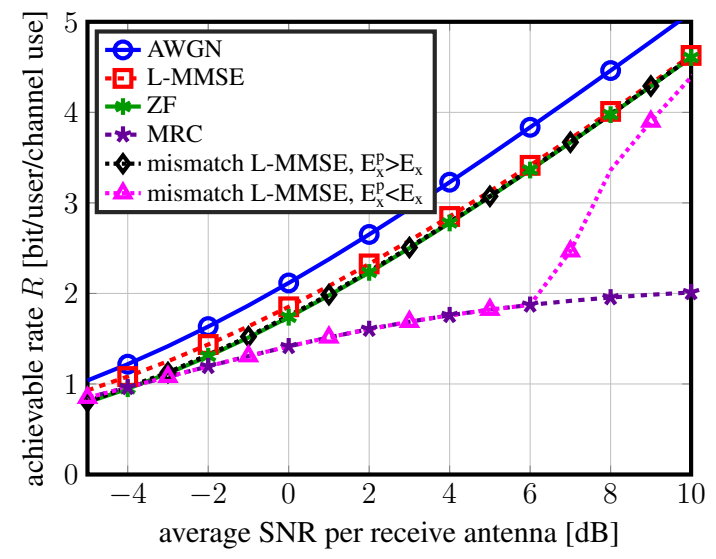

Fig. 2. Achievable rates for different equalizers with $\beta=0.3$. Overestimating $\left(E_{\mathrm{x}}^{\mathrm{p}}>E_{\mathrm{x}}\right)$ and underestimating $E_{\mathrm{x}}^{\mathrm{p}}<E_{\mathrm{x}}$ ) the signal power in the L-MMSE equalizer entails significant losses in terms of the achievable rates.

\section{L-MMSE with PARAMETER Mismatch}

Although L-MMSE significantly outperforms MRC and ZF equalization, it requires knowledge of the signal and noise powers. We now analyze the impact of a signal power parameter mismatch on the performance of the L-MMSE equalizer.

\section{A. Mismatch Analysis of AMP-based L-MMSE equalizer}

Analogously to [15, Sec. III-A], the mcB-AMP algorithm with a mismatched Gaussian prior $p\left(x_{u}\right)=\mathcal{C N}\left(0, E_{\mathrm{x}}^{\mathrm{p}}\right)$, $\forall u$, achieves the performance of a L-MMSE equalizer with mismatched signal power defined by $E_{\mathrm{x}}^{\mathrm{p}}$. The following lemma provides the SE equation for this mismatched L-MMSE algorithm; the proof can be established from the results of the coupled SE equations in [15, Thm. 1]:

Lemma 4. Fix $\beta$ and let $\mathbf{H}$ have uniform channel gains. Then, in the large-antenna limit, the performance of an optimally tuned AMP-based L-MMSE estimator with a mismatched signal power $E_{\mathrm{x}}^{\mathrm{p}}$ is given by the following coupled SE equations:

$$
\sigma_{t+1}^{2}=N_{0}+\beta \frac{\theta_{t}^{4} E_{\mathrm{x}}+E_{\mathrm{x}}^{\mathrm{p}} \sigma_{t}^{2}}{\left(E_{\mathrm{x}}^{\mathrm{p}}+\theta_{t}^{2}\right)^{2}} \quad \text { and } \theta_{t+1}^{2}=N_{0}+\beta \frac{E_{\mathrm{x}}^{\mathrm{p}} \theta_{t}^{2}}{E_{\mathrm{x}}^{\mathrm{p}}+\theta_{t}^{2}} \text {. }
$$

We note that for $t \rightarrow \infty$ and in the large-antenna limit, the result from Lemma 4 empirically matches the performance of the standard L-MMSE estimator in Section II-B with $N_{0} / E_{\mathrm{x}}^{\mathrm{p}}$. For no parameter mismatch, i.e., for $E_{\mathrm{x}}^{\mathrm{p}}=E_{\mathrm{x}}$, we have:

Corollary 5. Let $E_{\mathrm{x}}=E_{\mathrm{x}}^{\mathrm{p}}$. Then, the $S E$ equations in Lemma 4 for $t \rightarrow \infty$ coincide with the Tse-Hanly equation in [5, Thm. 3.1] for the L-MMSE equalizer with SIR $=1 / \sigma^{2}$.

\section{B. Numerical Analysis of Signal Power Mismatch}

Fig. 2 illustrates the impact of parameter mismatch on the achievable rate of L-MMSE equalization in a MIMO system with an antenna ratio of $\beta=0.3$. The dotted black and magenta curves show the achievable rate of the mismatched L-MMSE equalizer with overestimated and underestimated signal powers. The mismatched signal power $E_{\mathrm{x}}^{\mathrm{p}}$ is set to the 90-percentile of a signal-power estimator with two training data symbols. In words, for a given SNR, the mismatched L-MMSE equalizer will have an achievable rate between that of the exact L-MMSE equalizer (red curve) and the minimum 
of the mismatched versions (black and magenta dotted curves) in $90 \%$ of the transmissions. Clearly, the mismatched L-MMSE equalizer may experience a significant rate loss. We also see that underestimating the signal power $\left(E_{\mathrm{x}}^{\mathrm{p}}<E_{\mathrm{x}}\right)$ results in a performance close to that of MRC; overestimating $\left(E_{\mathrm{x}}^{\mathrm{p}}>E_{\mathrm{x}}\right)$ results in a performance close to that of the $\mathrm{ZF}$ equalizer.

\section{NOPE: NONPARAMETRIC EQUALIZER}

To cope with the detrimental effects of parameter mismatches in the L-MMSE equalizer, we now present our NOPE algorithm, which does not require knowledge of the signal or noise powers.

\section{A. The NOPE Algorithm}

MMSE-AMP, as in Algorithm 1, requires knowledge of the true transmit signal prior distribution $p(\mathbf{x})$ and the signal power $E_{\mathrm{x}}$. To design a nonparametric version that does not need this information, we need to solve the following issues:

- NOPE will not have any knowledge of the true signal prior. Hence, to tune the threshold parameter $\tau^{t}$ in (1), we need a way to estimate the MSE function $\Psi\left(\tilde{\sigma}_{t}^{2}, \tau^{t}\right)$.

- The signal power $E_{\mathrm{x}}$ must be tuned in each algorithm iteration $t$ to achieve optimal performance

Reference [21] develops a nonparametric approach to tune the threshold parameter of AMP-based sparse signal recovery using Stein's unbiased risk estimate (SURE) [22]. Inspired by this approach, we will tune both the (unknown) signal power $E_{\mathrm{x}}$ and the parameter $\tau^{t}$ using SURE. We start by defining the parameter $\gamma^{t}=E_{\mathrm{x}} / \tau^{t}$ and rewrite the functions $\mathrm{F}^{\mathrm{mm}}\left(x_{\ell}, \gamma^{t}\right)=\frac{\gamma^{t}}{\gamma^{t}+1} x_{\ell}$ and $\mathrm{F}^{\prime \mathrm{mm}}\left(x_{\ell}, \gamma^{t}\right)=\frac{\gamma^{t}}{\gamma^{t}+1}$ in Algorithm 1. As a consequence of this parameter change, we only need to estimate a single parameter per iteration, namely $\gamma^{t}$.

Optimal tuning within NOPE can be achieved if the parameters $\left\{\gamma^{1}, \ldots, \gamma^{t_{\max }}\right\}$ are tuned so that the MSE function $\Psi$ is minimized at iteration $t_{\max }$. As shown in [15, Thm. 3] a joint optimization over all parameters is not required-instead optimal tuning can be achieved by tuning each parameter $\gamma^{t}$ separately at iteration $t$ starting from $t=1$ to $t_{\text {max }}$.

To estimate the MSE function $\Psi$ without knowledge of the true signal prior, we use the SURE function, which is given by

$$
\begin{aligned}
\hat{\Psi}\left(\tilde{\sigma}_{t}^{2}, \gamma^{t}\right)= & \frac{1}{U}\left\|\mathrm{~F}^{\mathrm{mm}}\left(\mathbf{z}^{t}, \gamma^{t}\right)-\mathbf{z}^{t}\right\|^{2} \\
& +\tilde{\sigma}_{t}^{2}+2 \tilde{\sigma}_{t}^{2}\left\langle\mathrm{~F}^{\prime \mathrm{mm}}\left(\mathbf{z}^{t}, \gamma^{t}\right)-1\right\rangle \\
= & \tilde{\sigma}_{t}^{2} \frac{\gamma^{t}-1}{\gamma^{t}+1}+\frac{\left\|\mathbf{z}^{t}\right\|_{2}^{2}}{U\left(\gamma^{t}+1\right)^{2}} .
\end{aligned}
$$

The minimum of the SURE function is achieved for $\gamma_{\min }^{t}=$ $\left\|\mathbf{z}^{t}\right\|_{2}^{2} /\left(U \tilde{\sigma}_{t}^{2}\right)-1$. Hence, we replace the tuning stage in (1) by $\gamma_{\min }^{t}$ to arrive at the following algorithm we call NOPE:

Algorithm 2. Initialize $t=1, \mathbf{r}^{1}=\mathbf{y}$, and $\mathbf{x}^{1}=\mathbf{0}$. Then, for every iteration $t=1,2, \ldots, t_{\max }$ compute the following steps:

$$
\begin{aligned}
\mathbf{z}^{t} & =\mathbf{x}^{t}+\mathbf{H}^{\mathrm{H}} \mathbf{r}^{t} \\
\gamma^{t} & =\frac{1}{\beta} \frac{\left\|\mathbf{z}^{t}\right\|_{2}^{2}}{\left\|\mathbf{r}^{t}\right\|_{2}^{2}}-1 \\
\mathbf{x}^{t+1} & =\frac{\gamma^{t}}{\gamma^{t}+1} \mathbf{z}^{t} \\
\mathbf{r}^{t+1} & =\mathbf{y}-\mathbf{H} \mathbf{x}^{t+1}+\beta \mathbf{r}^{t} \frac{\gamma^{t}}{\gamma^{t}+1} .
\end{aligned}
$$

The following result establishes the fact that NOPE achieves the same performance as that of an L-MMSE equalizer that has perfect knowledge of the signal and noise powers.

Corollary 6. Let $\mathbf{H}$ have uniform channel gains. In the largeantenna limit and for $t \rightarrow \infty$, NOPE as in Algorithm 2 achieves the same SIR performance as the L-MMSE equalizer.

A sketch of the proof is as follows. By following the steps in [21, Sec. 4.4], we see that minimizing the SURE function (4) for the NOPE algorithm results in optimal tuning. Since optimal tuning is used, the SE equation from Lemma 1 is valid for NOPE and coincides to the SIR expression for the L-MMSE equalizer given by Tse and Hanly in [5, Thm. 3.1].

\section{B. Robust Implementation of NOPE}

NOPE, as in Algorithm 2, requires the channel matrix $\mathbf{H}$ to have uniform gains, which is rarely satisfied in practice. We next outline how NOPE can be made robust to more general channel matrices. We will use ideas from the generalized approximate message passing (GAMP) algorithm [23]. However, instead of allowing arbitrary variances in the channel matrix, we only assume that each user experiences a different variance (e.g., caused by large-scale fading). This assumption allows us to rewrite the channel matrix as $\mathbf{H}=\widetilde{\mathbf{H D}}$, where each element of $\widetilde{\mathbf{H}}$ is distributed $\mathcal{C N}(0,1 / B)$ and $\mathbf{D}$ is a diagonal matrix containing the users' individual gains. With this formulation, we can estimate the gain of the $\ell$-th user as $\hat{d}_{\ell}^{2}=\sum_{j=1}^{B}\left|H_{j, \ell}\right|^{2}$. Thus, $\mathbf{D}$ is estimated with a diagonal matrix $\hat{\mathbf{D}}$, where the $\ell$-th diagonal element is given by $\hat{d}_{\ell}$. By using GAMP [23], we first generalize Algorithm 1 to support nonuniform channel gains. The generalization requires us to modify the posterior mean function in (2) into an element-wise operation defined as

$$
\mathrm{F}_{\ell}^{\mathrm{mm}}\left(z_{\ell}^{t}, \tau^{t}\right)=\frac{E_{\mathrm{x}}}{E_{\mathrm{x}}+\tau^{t} / \hat{d}_{\ell}^{2}} z_{\ell}^{t} .
$$

Furthermore, step (3) in Algorithm 1 must be replaced by $\mathbf{r}^{t+1}=\mathbf{y}-\mathbf{H D}^{-2} \mathbf{x}^{t+1}+\beta \mathbf{r}^{t} \frac{1}{U} \sum_{\ell=1}^{U} \mathrm{~F}_{\ell}^{\prime m m}\left(z_{\ell}^{t}, \tau^{t}\right)$. We are now able to convert this generalized MMSE-AMP algorithm into a nonparametric algorithm by estimating the parameters $E_{\mathrm{x}}$ and $\tau^{t}$ in (5) in the large-antenna limit as given in the following result; the proof is given in Appendix A-B.

Theorem 7. In the large-antenna limit, the parameters $E_{\mathrm{x}}$ and $\tau^{t}$ in (5) can be estimated using

$$
\hat{E}_{\mathrm{x}}=\frac{\left\|\hat{\mathbf{D}}^{-1} \mathbf{z}^{t}\right\|_{2}^{2}-\beta\left\|\mathbf{r}^{t}\right\|_{2}^{2}}{\sum_{\ell=1}^{U} \hat{d}_{\ell}^{2}} \quad \text { and } \quad \hat{\tau}^{t}=\frac{1}{B}\left\|\mathbf{r}^{t}\right\|^{2} .
$$

Theorem 7 completes the necessary modifications to derive a robust version of the NOPE algorithm.

\section{Numerical Results and Conclusion}

Fig. 3 shows symbol error-rate (SER) simulation results in a $B=128$ and $U=96$ massive MU-MIMO system with QPSK modulation. We show the performance of $\mathrm{ZF}$ equalization and the AWGN lower bound as a fundamental performance limit. Evidently, the SER performance of NOPE with 20 iterations is virtually indistinguishable from the L-MMSE estimator, which requires exact knowledge of both the signal and noise powers. Thus, NOPE is suitable for situations in which the transmit 


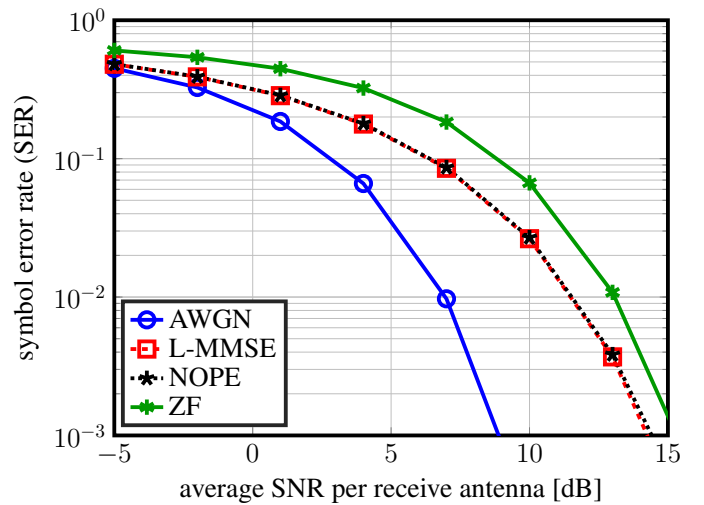

Fig. 3. Symbol error-rate of NOPE algorithm in a $128 \times 96$ massive MUMIMO system. NOPE closely approaches the performance of the L-MMSE estimator that requires exact knowledge of the signal and noise power.

constellation may be unknown to the receiver. In addition, NOPE often requires lower computational complexity than the L-MMSE estimator as it avoids the computation of a large matrix inversion, i.e., all involved operations for NOPE are matrix-vector products. These results demonstrate that largedimensionality of MU-MIMO systems provides the unique opportunity to design nonparametric algorithms that directly estimate the key system or model parameters from the received data, making them resilient to dynamic variations or model mismatches, while avoiding manual parameter tuning.

\section{APPENDIX A \\ PROOFS}

\section{A. Proof of Lemma 3}

Recall that the maximum achievable rate of IO-PME at SNR $E_{\mathrm{x}} / N_{0}$ is given by $C_{\mathrm{AWGN}}=\log _{2}\left(1+E_{\mathrm{x}} / N_{0}\right)$. In the large antenna limit, the maximum achievable rates for MRC, ZF and L-MMSE at SNR $E_{\mathrm{x}} / \hat{N}_{0}$ are given by $R=\log _{2}\left(1+E_{\mathrm{x}} / \sigma^{2}\right)$, where $\sigma^{2}$ is obtained from the following fixed-point equation:

$$
\sigma^{2}=\hat{N}_{0}+\beta^{\star} \Psi\left(\sigma^{2}\right),
$$

where $\Psi\left(\sigma^{2}\right)$ is $E_{\mathrm{x}}, \sigma^{2}$, and $\frac{E_{\mathrm{x}}}{E_{\mathrm{x}}+\sigma^{2}} \sigma^{2}$ for MRC, ZF and LMMSE, respectively [5]. Hence, the SNR loss is given by:

$$
\delta \mathrm{SNR}=\frac{N_{0}}{\hat{N}_{0}} \stackrel{(\text { a) }}{=} \frac{N_{0}}{\sigma^{2}-\beta^{\star} \Psi\left(\sigma^{2}\right)} \stackrel{\stackrel{(b)}{=}}{=}\left(1-\beta^{\star} \Psi\left(\frac{E_{\mathrm{x}}}{2^{R}-1}\right) \frac{2^{R}-1}{E_{\mathrm{x}}}\right)^{-1} .
$$

Here, (a) follows from (7), and (b) follows from the fact that since $R=C_{\mathrm{AWGN}}$, we have $\sigma^{2}=N_{0}=\frac{E_{\mathrm{x}}}{2^{R}-1}$. We can now extract $\beta^{\star}$ given $\delta$ SNR as given by Lemma 3. Moreover, for any $\beta \leq \beta^{\star}$ the SNR loss will be smaller than $\delta$ SNR.

\section{B. Proof of Theorem 7}

By the decoupling property of GAMP [23], each entry of the output $\mathbf{z}^{t}$ can be modeled as $z_{\ell}^{t}=\hat{d}_{\ell}^{2} x_{\ell}+\hat{d}_{\ell} w_{\ell}^{t}$ in the largeantenna limit, with $w_{\ell}^{t} \sim \mathcal{C N}\left(0, \sigma_{t}^{2}\right)$ where $\sigma_{t}^{2}$ is computed using the SE framework [23, Sec. V-C]. In the large-antenna limit, we estimate the signal power from the expression

$$
\begin{aligned}
\lim _{U \rightarrow \infty} \frac{1}{U}\left\|\hat{\mathbf{D}}^{-1} \mathbf{z}^{t}\right\|_{2}^{2} & \stackrel{(\mathrm{a})}{=} \lim _{U \rightarrow \infty} \frac{1}{U} \sum_{\ell=1}^{U} \mathbb{E}\left[\left|z_{\ell}^{t} / \hat{d}_{\ell}\right|^{2}\right] \\
& =\lim _{U \rightarrow \infty} \frac{1}{U} \sum_{\ell=1}^{U} \mathbb{E}\left[\left|w_{\ell}^{t}\right|^{2}\right]+\mathbb{E}\left[\left|x_{\ell}\right|^{2}\right] \hat{d}_{\ell}^{2} \\
& =\sigma_{t}^{2}+\hat{E}_{\mathrm{x}} \lim _{U \rightarrow \infty} \frac{1}{U} \sum_{\ell=1}^{U} \hat{d}_{\ell}^{2} .
\end{aligned}
$$

Here, (a) follows from Kolmogorov's strong law of large numbers [24] given that $\sum_{\ell=1}^{\infty}\left(E_{\mathrm{x}} d_{\ell}^{2}+\sigma^{2}\right) / \ell^{2}<\infty$, which is satisfied since the user gains $d_{\ell}^{2}$ are finite for all users. As shown in [18], the parameters $\tau^{t}$ and $\sigma_{t}^{2}$ in (8) can be estimated from $\left\|\mathbf{r}^{t}\right\|^{2} / B$. We can finally solve (8) for $\hat{E}_{\mathrm{x}}$ and obtain (6).

\section{REFERENCES}

[1] E. Larsson, O. Edfors, F. Tufvesson, and T. Marzetta, "Massive MIMO for next generation wireless systems," IEEE Commun. Mag., vol. 52, no. 2, pp. 186-195, Feb. 2014.

[2] J. Andrews, S. Buzzi, W. Choi, S. Hanly, A. Lozano, A. Soong, and J. Zhang, "What will 5G be?" IEEE J. Sel. Areas Commun., vol. 32, no. 6, pp. 1065-1082, Jun. 2014.

[3] S. Verdú, Multiuser Detection, 1st ed. Cambridge Univ. Press, 1998.

[4] C. Studer, M. Wenk, and A. Burg, "MIMO transmission with residual transmit-RF impairments," in Int. ITG Workshop on Smart Antennas (WSA), Feb. 2010, pp. 189-196.

[5] D. Tse and S. Hanly, "Linear multiuser receivers: effective interference, effective bandwidth and user capacity," IEEE Trans. Inf. Theory, vol. 45, no. 2, pp. 641-657, Mar. 1999.

[6] U. Madhow and M. L. Honig, "MMSE interference suppression for direct-sequence spread-spectrum CDMA," IEEE Trans. Commun., vol. 42, no. 12 , pp. $3178-3188$, Aug. 1994.

[7] Z. Xie, R. T. Short, and C. K. Rushforth, "A family of suboptimum detectors for coherent multiuser communications," IEEE Journal on Sel. Areas in Commun., vol. 8, no. 4, pp. 683-690, Aug. 1990.

[8] S. Verdú and S. Shamai, "Spectral efficiency of CDMA with random spreading," IEEE Trans. Inf. Theory, vol. 45, no. 2, pp. 622-640, Mar. 1999.

[9] K. R. Kumar, G. Caire, and A. L. Moustakas, "Asymptotic performance of linear receivers in MIMO fading channels," IEEE Trans. Inf. Theory, vol. 55, no. 10, pp. 4398-4418, Oct. 2009.

[10] S. Shamai and S. Verdú, "The impact of frequency-flat fading on the spectral efficiency of CDMA," IEEE Trans. Inf. Theory, vol. 47, no. 4, pp. 1302-1327, May 2001.

[11] J. Hoydis, S. ten Brink, and M. Debbah, "Massive MIMO: How many antennas do we need?" in Proc. Allerton Conf. Commun., Contr., Comput., Sept. 2011, pp. 545-550.

[12] N. Al-Dhahir and J. M. Cioffi, "Mismatched finite-complexity MMSE decision feedback equalizers," IEEE Trans. Signal Process., vol. 45, no. 4, pp. 935-944, Apr. 1997.

[13] M. Honig, U. Madhow, and S. Verdú, "Blind adaptive multiuser detection," IEEE Trans. Inf. Theory, vol. 41, no. 4, pp. 944-960, Jul. 1995.

[14] X. Wang and H. V. Poor, "Blind multiuser detection: A subspace approach," IEEE Trans. Inf. Theory, vol. 44, no. 2, pp. 677-690, Mar. 1998.

[15] C. Jeon, A. Maleki, and C. Studer, "On the performance of mismatched data detection in large MIMO systems," in Proc. IEEE Int. Symp. Inf. Theory (ISIT), Jul. 2016, pp. 180-184.

[16] D. L. Donoho, A. Maleki, and A. Montanari, "How to design message passing algorithms for compressed sensing," preprint, 2011.

[17] C. Studer, S. Fateh, and D. Seethaler, "ASIC implementation of softinput soft-output MIMO detection using MMSE parallel interference cancellation," IEEE J. Solid-State Circuits, vol. 46, no. 7, pp. 1754-1765, Jul. 2011

[18] A. Montanari, Graphical models concepts in compressed sensing, Compressed Sensing (Y.C. Eldar and G. Kutyniok, eds.). Cambridge Univ. Press, 2012.

[19] J. Hoydis, S. Ten Brink, and M. Debbah, "Massive MIMO in the UL/DL of cellular networks: How many antennas do we need?" IEEE J. Sel. Areas Commun., vol. 31, no. 2, pp. 160-171, Feb. 2013.

[20] D. Guo and S. Verdú, "Randomly spread CDMA: Asymptotics via statistical physics," IEEE Trans. Inf. Theory, vol. 51, no. 6, pp. 19832010, Jun. 2005.

[21] A. Mousavi, A. Maleki, and R. G. Baraniuk, "Consistent parameter estimation for LASSO and approximate message passing," arXiv preprint: 1511.01017 [math.ST], Nov. 2015.

[22] C. M. Stein, "Estimation of the mean of a multivariate normal distribution," The Annals of Statistics, pp. 1135-1151, 1981.

[23] S. Rangan, "Generalized approximate message passing for estimation with random linear mixing," IEEE Int. Symp. Inf. Theory (ISIT), Aug. 2011.

[24] P. K. Sen and J. M. Singer, "Large sample methods in statistics: an introduction with applications," Champion and Hall, 1993. 